\title{
Síntesis de una Matriz Cuasiceramica Destinada a la Obtención de Fundentes Aglomerados para Proceso SAW y Evaluación Cinético-termodinámica de su Proceso de Humectación
}

\author{
(Synthesis of a Cuasiceramic Matrix destined to the Obtaining of Agglomerated Fuxes for the SAW Process and Kinetic and \\ Thermodynamic Evaluations of its Humectation Process)
}

\author{
Rafael Quintana-Puchol ${ }^{1}$,Omar Prieto-Garcia², Jesús E. Hernández-Ruiz ${ }^{3}$,Amado Cruz-Crespo ${ }^{1}$,Carlos R. Gómez-Pérez', Lorenzo \\ Perdomo-González \\ ${ }^{1}$ Centro de Investigaciones de Soldadura, Universidad Central de las Villas, Cuba $;{ }^{2}$ Dpto. de Química de la Facultad de Química y \\ Farmacia, Universidad Central de las Villas, Cuba; ${ }^{3}$ Dpto. de Física, Universidad Central de las Villas, Cuba

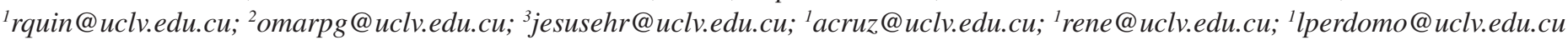

\section{Resumen}

En el presente trabajo se expone una concepción de la síntesis por sinterización, a relativa baja temperatura, de una matriz cuasicerámica amorfa destinada a la confección de fundentes aglomerados para el proceso SAW. Para diseñar la matriz cuasicerámica se hace una valoración de la composición química de los concentrados minerales de feldespato, zeolita y calcita, así como de la relación $\mathrm{SiO}_{2} / \mathrm{Al}_{2} \mathrm{O}_{3}$ de los alumosilicatos. También, como herramientas estratégicas para la síntesis, se usa el diagrama de fase $\mathrm{SiO}_{2}-\mathrm{Al}_{2} \mathrm{O}_{3}-\mathrm{CaO}$, los índices de basicidad y de actividad química y la temperatura Temman. Mediante valoración del espectro de DRX de la matriz y un novedoso criterio sobre el grado de sinterización se selecciona la variante de síntesis más adecuada. También se hace una valoración cinética y termodinámica del proceso de absorción isotérmica de la humectación por los granos de la matriz. El modelo de las isotermas de Langmuir se ajusta aceptablemente para describir el proceso de absorción de humedad por los granos de la matriz. La determinación de los valores de las constantes de las isotermas de Langmuir a diferentes humedades relativas y temperaturas permitió calcular los valores de entalpía, entropía y energía libre de Gibbs del proceso de adsorción.

Palabras claves: Síntesis de matriz;; proceso SAW; cinética y termodinámica de la humectación.

Astract: In the this paper is presented a conception of the synthesis by sinterizacion at relative low temperature, of an amorphous cuasiceramic matrix to be used in agglomerated flux in the SAW process. In the design of the cuasiceramic it was made a valuation of the chemical composition of the diferent mineral concentrates (feldspar, zeolite and calcite), as well as of the relationship $\mathrm{SiO}_{2} / \mathrm{Al}_{2} \mathrm{O}_{3}$ of the alumosilicates. Also, as strategic tools for synthesis were used the phase diagram $\mathrm{SiO}_{2}-\mathrm{Al}_{2} \mathrm{O}_{3}-\mathrm{CaO}$, the basicity and chemical activity indexes and the Temman temperature. It was selected the more appropriate synthesis variant by a valuation of the DRX spectrum of the matrix and a new approach (criterion) on the sinterization grade. Kinetic and thermodynamic valuations of the isothermic absorption process of the humidity (moisture) by the matrix grains are also made. The Langmuir isotherm model was adjusted acceptably to describe the humidity adsorption process by matrix grains. The determination of the values of Langmuir isotherm constants to different relative humidities and temperatures allowed to calculate the values of entalpía, entropy and Gibbs free energy of the adsorption process.

Key-words: Matrix Synthesis; SAW process; kinetics and thermodynamics of the humectación.

\section{Introducción}

A escala planetaria, la soldadura automática por arco sumergido (SAW) representa alrededor del 6,9\% de todos los procesos empleados en las soldaduras donde interviene el arco eléctrico como fuente energética [1] y en Cuba representa el 5 $\%$ [2]. Entre las tareas que se ejecutan empleando la soldadura automática (SAW) se encuentra el recargue superficial destinado a la recuperación de piezas de alto valor agregado.

(Recebido em 13/02/2010; Texto final em 28/05/2010).
Este proceder, usado desde el surgimiento mismo de la soldadura automática, no es exclusivo de países emergentes, sino que también es ampliamente empleado en países con un elevado desarrollo industrial, tal como lo describen PROBST [1] y QUINTANA [2].

Un fundente aglomerado aleado destinado a la soldadura automática (SAW) está constituido por dos partes fundamentales, la matriz $(65-85 \%)$ y la carga aleante (5 - 25\%), que se unen mediante un aglomerante (7 - $10 \%$ en estado ya calcinado, comúnmente los silicatos de sódico y potasio) durante un proceso de peletización [2]. Estos tres elementos componentes inciden en el desempeño tecnológico y metalúrgico del fundente durante el proceso SAW [3]. 
La matriz de un fundente debe ser lo más homogénea en las fases que la constituyen con el objetivo de que no haya durante la soldadura reacciones topoquímicas, las que pueden provocar puntualmente heterogeneidad en las características físicomecánica y químicas del cordón de soldadura[4]. Una forma de obtener una matriz cuasicerámica lo más homogénea posible es sinterizarla con alto grado de amorfismo para que la superficie de los granos aporte campos de fuerza aproximadamente equivalentes. Otro aspecto a considerar, es que los silicatos de sodio y potasio, comúnmente empleados como aglutinantes, son higroscópicos y a temperaturas algo superior a 450 ${ }^{\circ} \mathrm{C}$ expulsa casi irreversiblemente la mayor parte del agua químicamente enlazada [5], la que en estado gaseoso, a partir de $2000{ }^{\circ} \mathrm{C}$, empieza a descomponerse por la acción del arco eléctrico en hidrógeno y oxígeno atómicos, que presentan una alta solubilidad en el acero líquido [6]. Al solidificarse el cordón de soldadura, queda atrapada una parte del hidrógeno atómico, que al recombinarse conforma la molécula $\mathrm{H}_{2}$, provocando la formación de poros, grietas y tensiones en el metal depositado, mientras el oxígeno reacciona con los componentes del acero produciendo inclusiones endógenas, defectos que disminuyen apreciablemente las propiedades mecánicas del cordón [6, 7].

Por ello, durante el desarrollo de fundentes para soldadura se ha de tener muy en cuenta que la matriz sea de baja higroscopicidad, para no afectar más allá de los límites permisibles la ya comprometida capacidad higroscópica del fundente, resultante de la aglomeración de la matriz con este tipo de silicatos en estado líquido. Se estima que contenidos de humedad superiores a $0,1 \%$ en las matrices de estos consumibles para la SAW pueden provocar efectos negativos sobre el cordón de soldadura [3, 7].

Sin dudas, sobre la matriz aglomerada recaen con mayor responsabilidad las propiedades tecnológicas y operacionales del proceso SAW, que se enmarcan fundamentalmente en la estabilidad del arco, desprendimiento de la escoria y, también, en las propiedades físico-térmicas de la escoria, como son la permeabilidad a los gases, densidad, la tensión superficial y la viscosidad; estas dos últimas influyen en las dimensiones y aspecto del cordón [3, 6]. En este sentido se describe, que la composición química y fásica, la capacidad higroscópica y el contenido de agua estructural de la matriz de un fundente definen la calidad del desempeño tecnológico del proceso SAW e influyen activamente en las funciones metalúrgicas del fundente $[4,6,8]$. De este modo, la determinación de la capacidad de una matriz de adsorber humedad de la atmósfera es un requisito metalúrgico y tecnológico imprescindible para el desarrollo de fundentes aglomerados.

El presente artículo tiene el propósito de presentar la síntesis, a partir de concentrados minerales, de una matriz cuasicerámica destinada al desarrollo de fundentes aglomerados que son usados en la soldadura automática por arco sumergido (SAW), exponiendo el estudio cinético y termodinámico del proceso de humectación de los granos de la matriz.

En el apartado 2 se describe brevemente las consideraciones de obtención y caracterización de la matriz cuasicerámica, cuyo estudio cinético y termodinámico de la humectación se expone posteriormente en el apartado 3. En la presentación y discusión de los resultados se destaca el hecho de que esta matriz no reacciona con el agua líquida, por lo que se deduce que durante la adsorción y difusión de la humedad, la matriz se comporta como un substrato inerte que solo aporta el campo de fuerzas para la adsorción, conclusión esta que se infiere de los experimentos de la determinación conductividad eléctrica y del índice $\mathrm{pH}$ del agua bidestilada, que ya tuvo en contacto con los granos de la matriz en agitación. En tanto, el resultado más importante de la síntesis y del ensayo de humectación es obtener matrices que a humedades relativas $(\mathrm{Hr})$ en el entorno del $90 \%$ y temperaturas por encima de los $30{ }^{\circ} \mathrm{C} \mathrm{el} \mathrm{incremento}$ porcentual en masa humeda de la matriz es inferior a $0,1 \%$.

\section{Formulación y Síntesis de la matriz cuasicerámica}

La imposibilidad de contar con concentrados de materias primas clásicas idóneas de alta pureza indujo a sintetizar la matriz de un fundente aglomerado, a partir de rocas minerales beneficiadas sólo mediante procesos de trituración, tamizado y lavado, como una cuasicerámica amorfa buscando un alto grado de homogeneidad en la composición química y fásica en todo el cuerpo de la matriz. La matriz cuasicerámica se obtuvo por sinterización a relativa baja temperatura (alrededor de $900{ }^{\circ} \mathrm{C}$ ).

Para la confección de la matriz se concibió que la fuente de sílice y alúmina partiera de dos tipos de concentrados de rocas con minerales alumosilicáticos: una zeolítica (mineral principal: eulandita-clipnotilolita, $\approx 93 \%$ y otra feldespática (anortoclasa, $\approx 42 \%$ ) y rica en cuarzo. Las composiciones químicas de ambas rocas minerales beneficiadas se exponen en la Tabla 1.

A pesar de que las composiciones químicas de ambos concentrados son similares, los minerales principales de ambos presentan estructuras tectosilicáticas disímiles, que determinan su comportamiento durante la síntesis de la matriz.

Tabla 1. Composición química expresado en por cientos (\%) de los concentrados minerales de las rocas beneficiadas

\begin{tabular}{|c|c|c|c|}
\hline \multirow{2}{*}{ Óxidos } & Zeolita & Feldespato & Caliza \\
\cline { 2 - 4 } & $\%$ & $\%$ & $\%$ \\
\hline $\mathrm{SiO}_{2}$ & 66,05 & 76,13 & 0,73 \\
\hline $\mathrm{Al}_{2} \mathrm{O}_{3}$ & 11,15 & 12,67 & 0,23 \\
\hline $\mathrm{TiO}_{2}$ & 0,45 & 0,01 & 0,17 \\
\hline $\mathrm{Fe}_{2} \mathrm{O}_{2}$ & 1,75 & 0,38 & 0,17 \\
\hline $\mathrm{FeO}$ & 0,50 & 0,02 & - \\
\hline $\mathrm{MnO}$ & - & 0,01 & - \\
\hline $\mathrm{NiO}$ & - & 0 & 0,03 \\
\hline $\mathrm{MgO}$ & 0,50 & 0,01 & 0,65 \\
\hline $\mathrm{CaO}$ & 4,50 & 0,80 & 54,49 \\
\hline $\mathrm{Na}_{2} \mathrm{O}$ & 2,75 & 4,04 & - \\
\hline $\mathrm{K}_{2} \mathrm{O}$ & 1,00 & 2,68 & 0,46 \\
\hline $\mathrm{P}_{2} \mathrm{O}_{5}$ & - & 0,02 & - \\
\hline $\mathrm{CO}_{2}$ & - & - & 42,70 \\
\hline $\mathrm{H}_{2} \mathrm{O}+$ & 6,50 & 0,30 & - \\
\hline $\mathrm{H}_{2} \mathrm{O}-$ & 4,50 & 2,50 & - \\
\hline $\mathrm{Total}$ & 99,65 & 99,57 & 99,63 \\
\hline
\end{tabular}




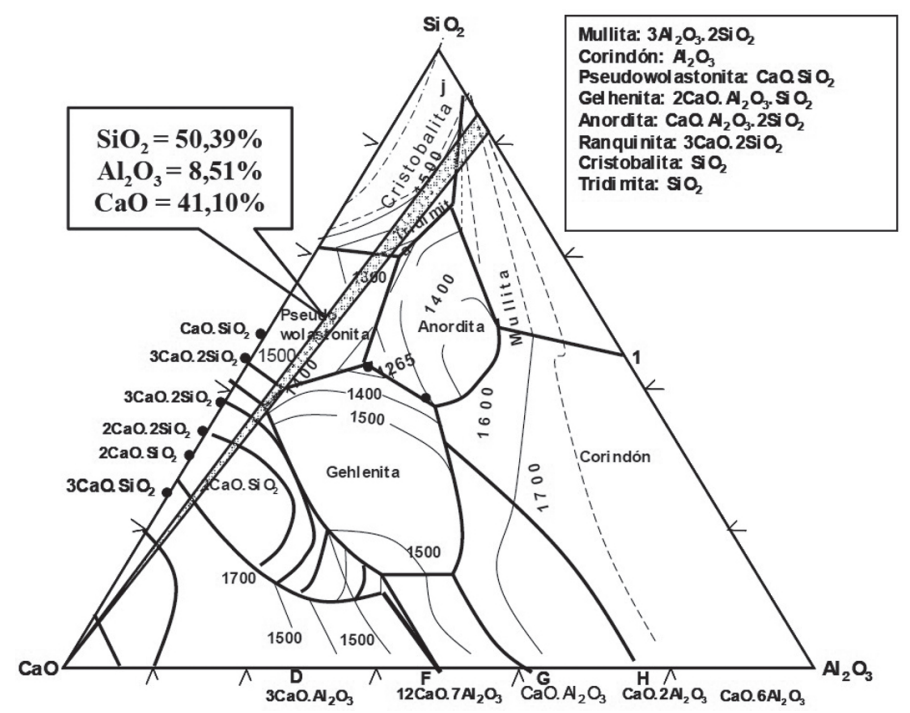

Figura 1. Representación en el diagrama de fase de la composición química más idónea de la matriz cuasicerámica a partir de la mezcla mineral: feldespato, zeolita y calcita [10].

La estructura tectosilicática de la zeolita es porosa, mientras que la del feldespato es compacta [9], características que definen las densidades de los concentrados determinados picnometricamente, cuyos valores son de 2,214 y $2,622 \mathrm{~g} / \mathrm{cm}^{3}$, respectivamente. El primer tipo de concentrado funge como un refractario, mientras que el segundo es un clásico agente fundente. Para diseñar la composición química de la matriz, que define sus comportamientos metalúrgico y tecnológico, se parte de las posibles mezclas de estos dos minerales que puedan brindar la más adecuada relación de sus tres óxidos principales: $\mathrm{SiO}_{2}, \mathrm{Al}_{2} \mathrm{O}_{3}$ y $\mathrm{CaO}$.

Cualquier combinación de ambos concentrados en una mezcla es rica en sílice (66-73\%) y el valor de $\mathrm{Al}_{2} \mathrm{O}_{3}$ oscila poco $(11,15$ y $12,67 \%)$, por lo que la relación $\mathrm{SiO}_{2} / \mathrm{Al}_{2} \mathrm{O}_{3}(5,92: 1$ para la zeolita y 5,65:1 para feldespato) inducen a que la composición resultante sólo pueda situarse en un estrecho segmento sobre el lado $\mathrm{SiO}_{2}-\mathrm{Al}_{2} \mathrm{O}_{3}$ del diagrama de fase ternario $\mathrm{SiO}_{2}-\mathrm{Al}_{2} \mathrm{O}_{3}-\mathrm{CaO}$, tal como se expone en la Figura 1.

Mediante la adición de $\mathrm{CaO}$ en forma de calcita $\left(\mathrm{CaCO}_{3}\right)$ se estrecha proporcionalmente dicho segmento desplazándose su punto medio en forma rectilínea hacia el vértice $\mathrm{CaO}$ atravesando varias zonas del diagrama ternario. Además es conocido que la calcita al descompone a $950{ }^{\circ} \mathrm{C}$ [9] hacen la función de agente fundente.

La zona idónea, desde el punto de vista metalúrgico para el diseño de una matriz, es aquella que, al ser atravesada por esa línea recta, sitúa uno de sus puntos entre las isotermas de 1300 y $1400{ }^{\circ} \mathrm{C}$ de la zona de la seudowollastonita y cuya composición es $50,39 \%$ de $\mathrm{SiO}_{2}, 8,51 \%$ de $\mathrm{Al}_{2} \mathrm{O}_{3}$ y $41,10 \%$ de $\mathrm{CaO}$ [4]. Este punto representa viscosidades $(\eta)$ entre 6 y 8 Pa.s, tensiones superficiales $(\sigma)$ entre 440 y $460 \mathrm{mN} / \mathrm{m}$ y densidades $(\rho)$ entre 2,537 y $2,517 \mathrm{~g} / \mathrm{cm}^{3}$ [5]. A partir de estos criterios se confecciona una mezcla mineral constituida por $27,52 \%$ de feldespato, 21,49 $\%$ de zeolita y $50,99 \%$ de caliza [11], que permite obtener una masa fundida, situada entre las referidas isotermas y conforma una composición química (Tabla 2), que augura una "prematriz" en estado fundido con propiedades aceptables.

Tabla 2. Composición química calculada de la mezcla de feldespato, zeolita y calcita calcinada a $950{ }^{\circ} \mathrm{C}$, así como los valores de

\begin{tabular}{|c|c|c|c|c|c|c|c|c|c|c|c|c|}
\hline Óxidos & $\mathrm{SiO}_{2}$ & $\mathrm{Al}_{2} \mathrm{O}_{3}$ & $\mathrm{CaO}$ & $\mathrm{Fe}_{2} \mathrm{O}_{3}$ & $\mathrm{FeO}$ & $\mathrm{NiO}$ & $\mathrm{MgO}$ & $\mathrm{TiO}_{2}$ & $\mathrm{P}_{2} \mathrm{O}_{5}$ & $\mathrm{Na}_{2} \mathrm{O}$ & $\mathrm{K}_{2} \mathrm{O}$ & $\sum$ \\
\hline $\mathrm{m}-\%$ & 47,54 & 8,03 & 38,78 & 0,76 & 0,15 & 0,02 & 0,59 & 0,25 & 0,01 & 2,28 & 1,59 & 100 \\
\hline \multicolumn{10}{|c|}{ Índice de Basicidad $\left(\mathrm{IB}_{\mathrm{m}}\right)=0,75$; Actividad química $\left(\mathrm{A}_{\mathrm{q}}\right)=0,68$} \\
\hline
\end{tabular}

A partir de la composición química expuesta (Tabla 2) se calcula el índice de basicidad de la posible matriz (IB ${ }_{\mathrm{m}}$ ), así como su la actividad química relativa $\left(A q_{r}\right)$ según las expresiones (1) y (2) desarrolladas por POTAPOV [12] partir de la propuesta por TULIANI [13]:

$$
\begin{aligned}
& I B_{m}=\frac{0,018 \mathrm{CaO}+0,025 \mathrm{MgO}+0,006 \mathrm{CaF}_{2}+0,014\left(\mathrm{Na}_{2} \mathrm{O}+\mathrm{K}_{2} \mathrm{O}+0,007(\mathrm{FeO}+\mathrm{MnO})\right.}{0,017 \mathrm{SiO}_{2}+0,005\left(\mathrm{Al}_{2} \mathrm{O}_{3}+\mathrm{TiO}_{2}+\mathrm{ZrO}_{2}\right)} \\
& A q_{r}=\frac{\left(\mathrm{SiO}_{2}\right)+0,5\left(\mathrm{TiO}_{2}\right)+0,4\left(\mathrm{Al}_{2} \mathrm{O}_{3}+\mathrm{ZrO}_{2}\right)+0,42\left(\mathrm{IB}_{m}\right)^{2}(\mathrm{MnO})}{100 \cdot \mathrm{IB}_{m}}
\end{aligned}
$$

En las ecuaciones (1) y (2) las fórmulas químicas expresan sus contenido en por ciento de la Tabla 2. Según los valores de los índices $\mathrm{IB}_{\mathrm{m}}=0,75$ y $A_{q}=0,68$ calculados (Tabla 2) se puede clasificar a la "prematriz propuesta" de ligeramente ácida (LA) y de altamente activa, aspectos conjugados que no favorecen de manera eficaz el afino y la actividad metalúrgica de la matriz diseñada [3, 14]. Para mejorar la actividad metalúrgica se puede incrementar la basicidad, tratando de reducir las dimensiones de los bloques estructurales $\left[\mathrm{Si}_{\mathrm{n}} \mathrm{O}_{2 \mathrm{n}}\right]^{\mathrm{z}-}$ de la matriz fundida $\mathrm{y}$, por tanto, disminuyendo su viscosidad y el posible contenido del ion oxígeno $\left[\mathrm{O}^{2-}\right]$ en la escoria resultante al estado líquido o líquidopastoso para una temperatura dada. La búsqueda de tal óxido tiene que conjugarse con toda la estrategia del sistema inicial de óxidos.

Al confeccionar la mezcla de polvos minerales es imprescindible someterla a un proceso de homogenización fásica (amorfismo) por calcinación $\left(<1100{ }^{\circ} \mathrm{C}\right) \mathrm{y}$, además, lograr solidificar, con cierta consistencia, esa mezcla de polvos 
topoquímicamente heterogénea mediante la unión de puentes de adherencia entre las partículas. Estos puentes pueden estar promovidos por una sustancia que funda, a cierta temperatura temprana, aglomerando determinada cantidad de sustancia superficial de los granos en contacto y difundiéndose dentro del grano, provocando, generalmente, una contracción del volumen y una mayor consistencia y homogeneidad del cuerpo de los granos "sinterizados".

La temperatura de "sinterización" mínima, a la que los granos sueltos (móviles) de la mezcla de polvos se unen dando al cuerpo de cierta consistencia, se denomina temperatura de Temman $\left(\mathrm{T}_{\mathrm{T}}\right)$ que está relacionada con la temperatura de fusión $\left(\mathrm{T}_{\mathrm{f}}\right)$ de las sustancias y el tiempo de exposición a una determinada temperatura: para metales $\mathrm{T}_{\mathrm{T}}=0,3 \ldots 0,4 \cdot \mathrm{T}_{\mathrm{f}}$, para sales $\mathrm{T}_{\mathrm{T}}=0,57 \cdot \mathrm{T}_{\mathrm{f}}$ y para silicatos $\mathrm{T}_{\mathrm{T}}=0,8 \ldots 0,9 \cdot \mathrm{T}_{\mathrm{f}}[5]$.

La acción fundente de la calcita y el feldespato se manifiesta mediante las temperaturas de sinterización a 820 y $1100{ }^{\circ} \mathrm{C}$, respectivamente. Sin embargo, las cinéticas de la sinterización y la coalescencia de estas alternativas son lentas a ambas temperaturas para lograr un cierto grado de adhesión entre las partículas en un razonable tiempo, por lo que hay que emplear una sustancia que disminuya aun más la temperatura y además acelere el proceso de sinterización.

El $\mathrm{Na}_{2} \mathrm{O}$ es uno de los vitrificantes más activo y que más disminuye la temperatura de sinterización superficial entre granos, provocando también un notable ensanchamiento del intervalo de fusión del coeficiente de adherencia. En este caso, el suministro de $\mathrm{Na}_{2} \mathrm{O}$ se realiza en forma de carbonato anhidro: $\mathrm{Na}_{2} \mathrm{CO}_{3}\left(\mathrm{MM}=106 \mathrm{~g} / \mathrm{mol}, \rho=2,533 \mathrm{~g} / \mathrm{cm}^{3}\right)$. El carbonato de sodio (3a) se funde totalmente a $840{ }^{\circ} \mathrm{C}$ sin descomponerse (3b) y la presión parcial de $\mathrm{CO}_{2}$ a esa temperatura alcanza $8,8.10^{-8}$ atm acorde a la ecuación (3c):

$$
\begin{gathered}
\mathrm{Na}_{2} \mathrm{CO}_{3} \rightarrow \mathrm{Na}_{2} \mathrm{O}+\mathrm{CO}_{2} \uparrow(3 \mathrm{a}) ; \quad \Delta G=76850-35,96 \mathrm{~T} \\
\log \left(\mathrm{P}_{\mathrm{CO}_{2}}\right)=-16797 / \mathrm{T}+7,86 \quad \text { (3c) }
\end{gathered}
$$

Según la ecuación (3c) la presión parcial del $\mathrm{CO}_{2}$ de la natrita $\left(\mathrm{Na}_{2} \mathrm{CO}_{3}\right)$ a $853{ }^{\circ} \mathrm{C}$ sigue siendo imperceptible, pero se incrementa significativamente debido a su reactividad manifiesta a causa de la interacción líquido-sólido con la sílice de los silicatos:

$$
2 \text { “" } \mathrm{SiO}_{2(\mathrm{~s})} "+\mathrm{Na}_{2} \mathrm{CO}_{3(1)} \mathrm{Na}_{2} \mathrm{Si}_{2} \mathrm{O}_{5}(\mathrm{l})+\mathrm{CO}_{2} \uparrow(4)
$$

Teniendo en consideración el efecto endotérmico principal del ATD (Figura 2) y la ecuación (4) se puede afirmar que esta reacción ocurre a temperaturas inferiores a la temperatura de fusión de la natrita. También hay que tener en cuenta que la mezcla de polvos presenta poros intergranular, pero la zeolita, uno de sus componentes, presenta microporos estructurales [15]. Es conocido que, desde el punto de vista de la termodinámica, la disminución de la energía libre superficial de una mezcla de polvos se realiza mediante dos vías: la sinterización y la coalescencia [16].

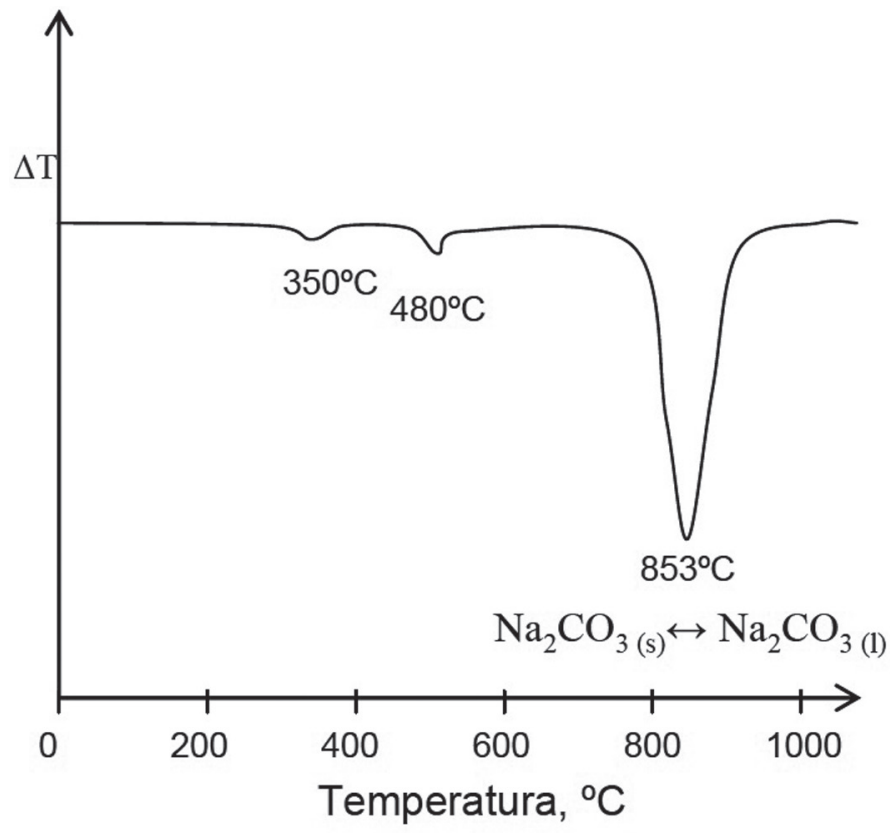

Figura 2. ATD de la fusión de $\mathrm{Na}_{2} \mathrm{CO}_{3}$

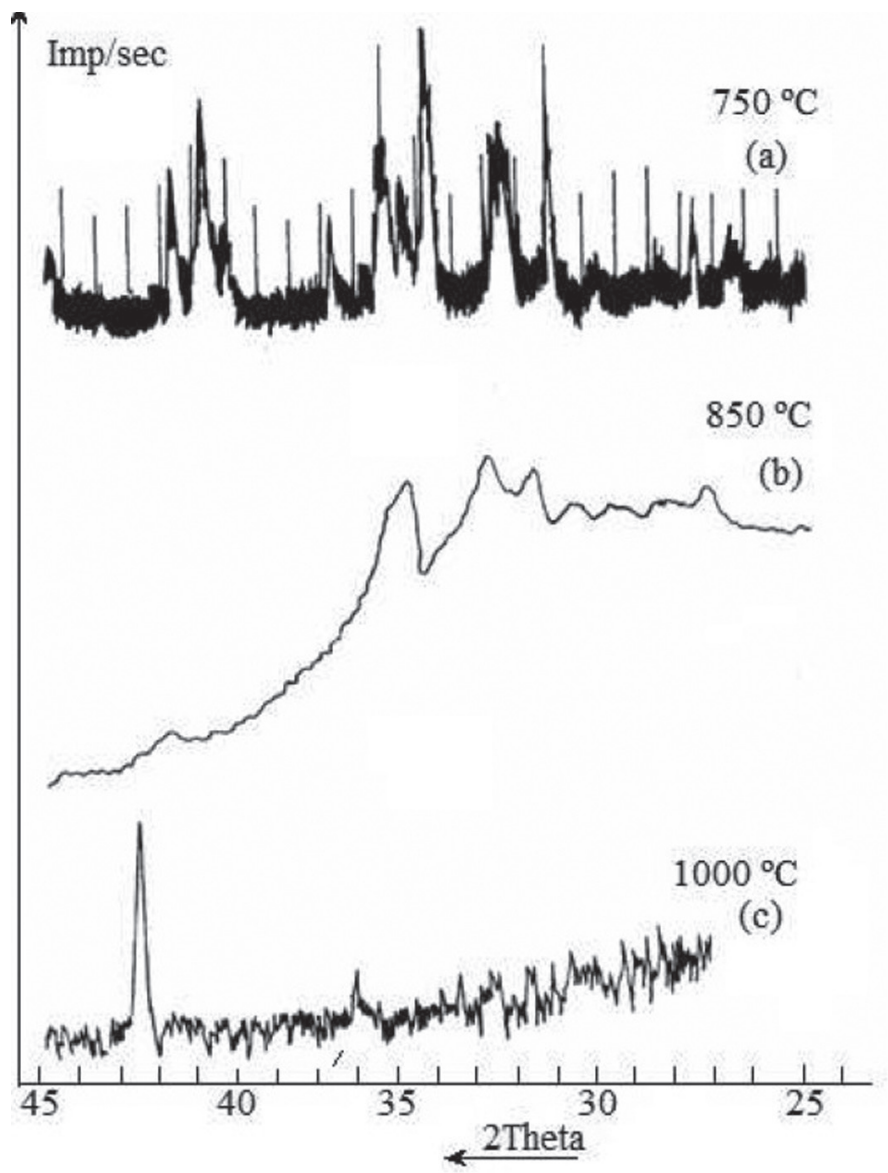

Figura 3. Difractogramas de las masas calcinadas a deferentes temperaturas

En el presente trabajo, durante el tratamiento térmico, la sinterización y la coalescencia ocurren simultáneamente, pero la velocidad de sinterización debe ser mayor que la de la 
Tabla 3. Datos iniciales para la determinación del grado de sinterización (GS) y sus valores, según (5)

\begin{tabular}{|c|c|c|c|c|c|c|c|c|c|c|c|c|c|}
\hline \multirow{3}{*}{$\mathrm{N}^{\mathrm{o}}$} & \multirow{3}{*}{$\mathrm{T},{ }^{\circ} \mathrm{C}$} & \multicolumn{3}{|c|}{ Masa inicial } & \multicolumn{3}{|c|}{ Pérdida en $\mathrm{HCl}$, g } & \multicolumn{3}{|c|}{ Valores de $\mathrm{pH}_{\mathrm{i}}$} & \multicolumn{3}{|c|}{ Grado de Sinterización, \% } \\
\hline & & $\mathrm{M}_{1}$ & $\mathrm{M}_{2}$ & $\mathrm{M}_{3}$ & $\mathrm{P}_{1}$ & $\mathrm{P}_{2}$ & $\mathrm{P}_{3}$ & $\mathrm{pH}_{1}$ & $\mathrm{pH}_{2}$ & $\mathrm{pH}_{3}$ & $\mathrm{GS}_{1}$ & $\mathrm{GS}_{2}$ & $\mathrm{GS}_{3}$ \\
\hline & & $2 \mathrm{~h}$ & $3 \mathrm{~h}$ & $4 \mathrm{~h}$ & $2 \mathrm{~h}$ & $3 \mathrm{~h}$ & $4 \mathrm{~h}$ & $2 \mathrm{~h}$ & $3 \mathrm{~h}$ & $4 \mathrm{~h}$ & $2 \mathrm{~h}$ & $3 \mathrm{~h}$ & $4 \mathrm{~h}$ \\
\hline 1 & 750 & 77,169 & 74,813 & 74,737 & 12,427 & 11,431 & 10,790 & 13,4 & 13,2 & 13 & 36,13 & 37,75 & 39,41 \\
\hline 2 & 800 & 70,117 & 69,758 & 70,737 & 5,292 & 6,287 & 6,714 & 11,2 & 10,9 & 10,1 & 54,95 & 55,21 & 59,82 \\
\hline 3 & 850 & 67,862 & 66,914 & 67,122 & 4,095 & 3,693 & 3,355 & 8,3 & 8,1 & 8 & 78,30 & 80,90 & 82,50 \\
\hline 4 & 900 & 66,263 & 64,427 & 64,715 & 1,527 & 1,125 & 0,765 & 7,7 & 7,5 & 7,4 & 88,61 & 91,59 & 93,41 \\
\hline 5 & 950 & 65,522 & 63,854 & 63,981 & 0,775 & 0,391 & 0,023 & 7,3 & 7,2 & 7,1 & 94,71 & 96,61 & 98,56 \\
\hline
\end{tabular}

Tabla 4. Composición química y valores de $\mathrm{IB}_{\mathrm{m}}$ y $\mathrm{A}_{\mathrm{q}}$ de la matriz cuasicerámicaobtenida a $950^{\circ} \mathrm{C}$ durante $4 \mathrm{~h}$

\begin{tabular}{|l|c|c|l|c|l|c|c|c|c|c|c|c|}
\hline Óxidos & $\mathrm{SiO}_{2}$ & $\mathrm{Al}_{2} \mathrm{O}_{3}$ & $\mathrm{Fe}_{2} \mathrm{O}_{3}$ & $\mathrm{TiO}_{2}$ & $\mathrm{FeO}$ & $\mathrm{MnO}$ & $\mathrm{NiO}$ & $\mathrm{MgO}$ & $\mathrm{CaO}$ & $\mathrm{Na}_{2} \mathrm{O}$ & $\mathrm{K}_{2} \mathrm{O}$ & $\mathrm{P}_{2} \mathrm{O}_{5}$ \\
\hline $\mathrm{m}-\%$ & 44.91 & 7.59 & 0.72 & 0.24 & 0.14 & 0.01 & 0.02 & 0.56 & 36.64 & 7.68 & 1.50 & 0.01 \\
\hline \multicolumn{10}{|c|}{ Índice de Basicidad $\left(I B_{m}\right)=0,80$ yctividad química relativa $\left(A_{q r}\right)=0,59$} \\
\hline
\end{tabular}

coalescencia, es decir que la disminución de la energía superficial se origina más rápidamente por la reducción del volumen total de los poros visibles y el crecimiento de granos (lo que provoca la contracción del material y la elevación de su densidad aparente) que por la disminución de la porosidad total a causa de la reducción de la superficie de los poros, tanto intergranulares como los estructurales al aumentar sus dimensiones.

A partir de todos estos criterios se añade $10 \%$ de natrita $\left(\mathrm{Na}_{2} \mathrm{CO}_{3}\right)$ a la formulación mineral de la mezcla inicial destinada para la confección final de la matriz por sinterización. A partir de esta formulación mineral en polvo $(<100 \mu \mathrm{m})$, se confeccionaron 15 briquetas verdes aglomeradas con una solución de alcohol polivinílico al $2 \%$, después de oreadas y precocidas a $450{ }^{\circ} \mathrm{C}$, se calcinaron a cinco diferentes temperaturas entre $750^{\circ} \mathrm{C}$ y 1000 ${ }^{\circ} \mathrm{C}$ durante 2,3 y 4 h (Tabla 3 ).

Las 15 briquetas cuasicerámicas se trituran cuidadosamente y por medio de tamizado se obtiene una masa granulada, cuyos granos se encuentran en el rango granulométrico entre $+0,2$ $\mathrm{mm}$ y $-2,5 \mathrm{~mm}$. Una parte de estos granos de cada briqueta se pulverizan para determinar el grado de amorfismo (GA), el grado de sinterización (GS) y la otra parte, sin pulverizar, se utiliza para la determinación de las características del proceso de humectación de los mismos.

Mediante difracción de rayos- $X$ se determina la temperatura, a la que la interacción entre los minerales en la masa de las briquetas proporciona un producto amorfo después del tratamiento térmico. En la Figura 3 se expone los difractogramas de la mezcla mineral tratadas a diferentes temperaturas durante 4h, observándose que a $850{ }^{\circ} \mathrm{C}$ se obtiene un producto con marcado GA. Se observó que entre 850 y $1000^{\circ} \mathrm{C}$, el tratamiento térmico puede transformar casi toda la estructura de los constituyentes alumosilicáticos de la mezcla mineral en amorfa, pero no alcanza a vitrificarse la masa de polvos, quedándose como una cerámica compacta pero de relativa baja consistencia mecánica, a la que se denominó cuasicerámica. Se determinó que a $950^{\circ} \mathrm{C}$ se obtiene el mayor grado de amorfismo (GA).

Para determinar el GS de la cuasicerámica se utiliza la fórmula (5) desarrollada por PORTAL [11], que se basa en el principio de conservación de la masa y el comportamiento acido-base del polvo $(<100 \mu \mathrm{m})$ de las cuasicerçamicas en agua y ante soluciones ácidas. Esta fórmula brinda criterios cuantitativos reproducibles en las mismas condiciones de síntesis sobre el estado de descomposición de los carbonatos y la existencia de óxidos alcalinos y alcalinos térreos libres, que no hayan reaccionado con la fase silicática a la temperatura de sinterización.

Donde $P_{\mathrm{i}}$ es la perdida de cierta cantidad de masa de polvo de $G S=\left(1-\left[\frac{P_{i}}{M_{i}}+\frac{p H_{i}-7}{p H_{i}}\right]\right) \cdot 100$

la cuasicerámica al ser atacada con una solución al 0,25 mol/L de $\mathrm{HCl}, \mathrm{M}_{\mathrm{i}}$ es la masa inicial de polvo de la cuasicerámica antes del ataque con ácido (Tabla 3), $\mathrm{pH}_{i}$ es el valor del logaritmo negativo de la concentración de $\mathrm{H}_{3} \mathrm{O}^{+}$a $25^{\circ} \mathrm{C}$ en $50 \mathrm{ml}$ de agua bidestilada que ya estuvo en agitación con $10 \mathrm{~g}$ de polvo de matriz durante $10 \mathrm{~min}$.

$\mathrm{Al}$ evaluar la posible interacción del agua destilada con el polvo $(<100 \mu \mathrm{m})$ de las matrices cuasicerámicas (Tabla 3), se pudo comprobar, que la fase líquida resultante de la agitación del polvo, de aquellas obtenidas a $950{ }^{\circ} \mathrm{C}$ y $4 \mathrm{~h}$, presentan los valores más adecuados y reproducibles, tanto para la conductividad eléctrica específica $\left(0,57 \cdot 10^{8} \Omega^{-1} \mathrm{~cm}^{-1}\right)$, como para el índice $p H$ $(7,1)$ que indican que no hubo prácticamente una interacción a considerar, es decir que a $950{ }^{\circ} \mathrm{C}$ y 4 h es la condición, a la que la cuasicerámica presenta el más alto grado de sinterización (GS) y la menor interacción con el agua bidestilada $(p H)$. Sin embargo, a $1000{ }^{\circ} \mathrm{C}$ y $2 \mathrm{~h}$ empieza a surgir de forma evidente fases cristalinas, que convierte a la matriz en una sustancia policomponente (Figura 3). Por lo que se puede considerar que el rango de los granos (entre $-0,25 \mathrm{~mm} \mathrm{y}+2,5 \mathrm{~mm}$ ) de esta matriz no reacciona con el agua y, en consecuencia, se comportan como un sustrato inerte que fundamentalmente aporta el campo de fuerzas y la topografía para la adsorción del vapor de agua del aire.

En la Tabla 4 se exponen el resultado del análisis químico obtenido por fluorescencia de rayos- $X$ de la cuasicerámica sintetizada a $950{ }^{\circ} \mathrm{C}$ durante $4 \mathrm{~h}$ y los valores de $\mathrm{IB}_{\mathrm{m}}$ y $\mathrm{A}_{\mathrm{q}}$, que indican un mejor desempeño metalúrgico y tecnológico. 
Tabla 5. Valores de Ei del conjunto de los elementos de menor

Ei al del Fe $(7,902 \mathrm{eV})$ presentes en $100 \mathrm{~g}$ de matriz

\begin{tabular}{|l|c|c|c|c|}
\hline Elem. & $\mathrm{m}-\%$ & mole & $\mathrm{Ei}(\mathrm{eV})$ & $\mathrm{kJ}$ \\
\hline $\mathrm{K}$ & 1,25 & 0,03196 & 4,339 & 13,383 \\
\hline $\mathrm{Na}$ & 5,70 & 0,24779 & 5,134 & 122,761 \\
\hline $\mathrm{Ca}$ & 26,17 & 0,65429 & 6,111 & 385,842 \\
\hline $\mathrm{Ti}$ & 0,14 & 0,00295 & 6,862 & 1,955 \\
\hline $\mathrm{Mg}$ & 0,33 & 0,01394 & 7,644 & 10,280 \\
\hline $\mathrm{Suma}$ & 33,59 & 0,95093 & & 534,221 \\
\hline
\end{tabular}

Tabla 6: Evaluación de los parámetros tecnológicos de la deposición de cordones por SAW empleando la matiz cuasicerámica granulada (de $+0,25 \mathrm{~mm}$ a $-2,5 \mathrm{~mm}$ ) como fundente

\begin{tabular}{|c|c|c|c|}
\hline Parámetros a observar & $\begin{array}{l}\text { Valores y criterios sobre los } \\
\text { parámetros a considerar }\end{array}$ & Criterio cualitativo & Indicador cuantitativo \\
\hline Estabilidad del arco & $\pm 5 \mathrm{~A}$ & excelente & 4,8 \\
\hline Presencia de llamas, humos o gases & $\begin{array}{l}\text { Ausencia total de llamas y ligera presencia } \\
\text { de gases }\end{array}$ & muy bien & 4,6 \\
\hline Desprendimiento de la escoria & Escoria auto separable & muy bien & 4,6 \\
\hline Aspecto del cordón & Se perciben huellas o marcas & muy bien & 4,6 \\
\hline
\end{tabular}

Tabla 7: Resultados del análisis químico promedio del alambre-electrodo, chapa de acero y de los cordones de soldadura empleando granos de cuasicerámica

\begin{tabular}{|l|c|c|c|c|c|}
\hline \multirow{2}{*}{ Materiales empleados en el proceso SAW } & \multicolumn{5}{|c|}{ Composición química, \%-m } \\
\cline { 2 - 6 } & $\mathrm{C}$ & $\mathrm{Si}$ & $\mathrm{Mn}$ & $\mathrm{S}$ & $\mathrm{P}$ \\
\hline Alambre-Electrodo EL8K, $\varnothing=2 \mathrm{~mm}$ & 0,10 & 0,21 & 0,55 & 0,053 & 0,041 \\
\hline Chapa de acero AS36 & 0,17 & 0,20 & 0,56 & 0,051 & 0,043 \\
\hline Tercera capa depositada & 0,07 & 0,22 & 0,38 & 0,021 & 0,013 \\
\hline
\end{tabular}

También, al valorar el contenido de la Tabla 4, se observa que los tres óxidos el sistema $\mathrm{SiO}_{2}-\mathrm{Al}_{2} \mathrm{O}_{3}-\mathrm{CaO}$ representan $89,14 \%$ y los elementos, $\mathrm{K}, \mathrm{Na}, \mathrm{Ca}, \mathrm{Mg}$ y Ti, de relativa baja energía de iotización (Ei) constituyen el 33,59\%, que representan en su conjunto 0,95093 mol. Para ionizar esa cantidad de elementos químicos en $100 \mathrm{~g}$ de matriz se necesita $534,22 \mathrm{~kJ}$, energía superior en $11,81 \%$ a la que se necesitaría para ionizar una cantidad equivalente de átomos de $\mathrm{Na}(471,12 \mathrm{~kJ})$ de relativo bajo potencial de ionización, por lo que se augura una buena estabilidad del fundente en el arco al usar esta matriz en su confección (ver Tabla 5). El resultado del análisis químico de la Tabla 4 es similar al calculado a partir de los análisis químicos de los concentrados minerales, lo que indica que los criterios esgrimidos para diseñar químicamente la matriz son correctos.

\section{Características tecnológicas de la cuasicerámica granulada}

Según recomendaciones de QUINTANA $[4,18]$ se realiza una evaluación del comportamiento de las características tecnológicas y operacionales de la matriz cuasicerámica granulada (de $+0,25 \mathrm{~mm}$ a $-2,5 \mathrm{~mm}$ ) durante el proceso SAW. El depósito de los cordones de soldadura se realiza sobre planchas de acero A36 (ASTM) de $20 \mathrm{~mm}$ de espesor, $160 \mathrm{~mm}$ de longitud y $90 \mathrm{~mm}$ de ancho, empleando un alambre-electrodo EL8K (AWS-A5.17) de $2 \mathrm{~mm}$. En el régimen de soldadura se usa una corriente (Is) de $200 \mathrm{~A}$, una tensión de $\operatorname{arco}\left(\mathrm{U}_{\mathrm{a}}\right)$ que oscila entre $25 \mathrm{~V}$ y $26 \mathrm{~V}$ y una velocidad de soldadura $\left(\mathrm{v}_{\mathrm{s}}\right)$ de $0,75 \mathrm{~m} \cdot \mathrm{min}^{-1}$

Para evaluar los respectivos parámetros tecnológicos del proceso SAW durante la deposición del cordón, se constituye una comisión de cinco expertos, quienes vierten individualmente y en secreto los criterios (Tabla 6) cuantitativos (números enteros: $2, \ldots, 5)$ respecto a dichos parámetros después de finalizado el proceso, en función de criterios previamente establecidos.

La composición química promedio del alambre electrodo, de las planchas empleadas y la de la última capa, al depositar tres cordones superpuestos con el régimen descrito, se exponen en la Tabla 7. Durante el proceso de soldadura se observa para los granos de la cuasicerámica una excelente estabilidad de arco, ausencia total de llamas y humo, un buen desprendimiento de la escoria. Sobre la superficie del cordón se denota ligeramente huellas indicativas de los frentes de solidificación durante la soldadura.

En la estructura metalogáfica obtenidas de la zona central de la tercera capa del cordón de soldadura (Figura 4) se muestran un predominio fundamental de ferrita poligonal (PF), encontrándose también presente ferrita de segunda fase (FS), 
así como en la zona oscura es probable la presencia de ferrita acicular (AF) [19] [3]. Las distintas fases que esta constituida la estructura metalografica obtenida se corresponde con el contenido de carbono según la composición química del acero depositado reportada en la tabla 7, que indica un predominio de la fase ferrítica. El pequeño incremento de silicio $(0,01 \%)$ en el metal depositado se debe a que la matriz es ligeramente acida $\left(\mathrm{IB}_{\mathrm{m}}=0.80\right)$.

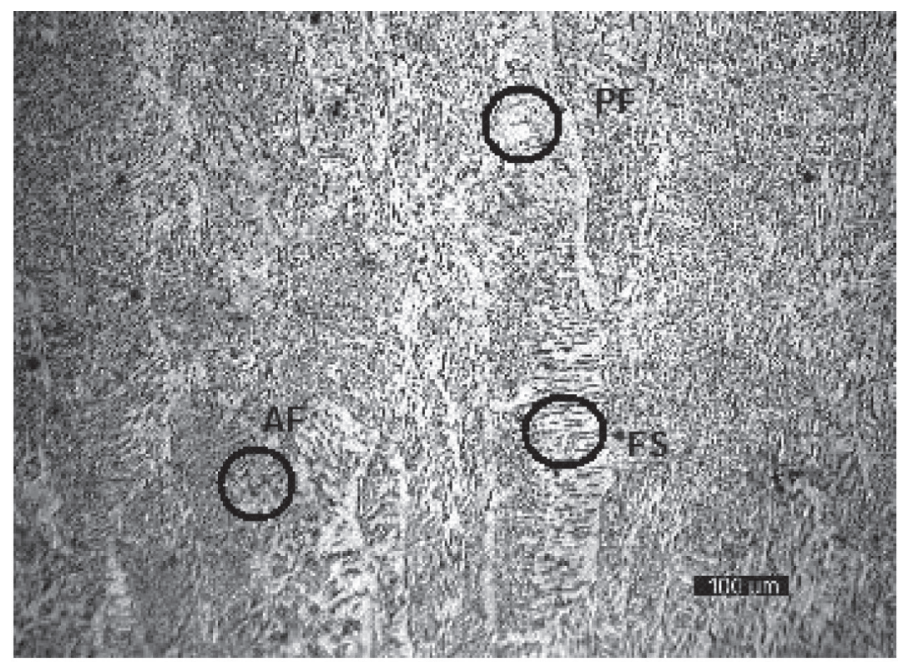

Figura 4. Estructura metalográfica del centro de la tercera capa del metal depositado con los granos de la cuasicerámica, sobre planchas de acero A36 (ASTM), de $20 \mathrm{~mm}$ x $160 \mathrm{~mm}$ x $90 \mathrm{~mm}$, con alambre-electrodo EL8K (AWS-A5.17), de $2 \mathrm{~mm}$. (Is $=200 \mathrm{~A}, 25 \mathrm{~V} \leq \mathrm{U}_{\mathrm{a}} \leq 26 \mathrm{~V} ; \mathrm{v}_{\mathrm{s}}=0,75 \mathrm{~m} / \mathrm{min}$ ). Ataque con Nital al $2 \%$.

La superficie de la escoria, que estuvo en contacto con el metal líquido, es vítrea, sin poros y refleja los detalles de la superficie del cordón, indicando que la temperatura de su solidificación es inferior a la del metal depositado. En todos los cordones, no se detectan grietas, ni poros. La calificación $(4,65 \pm 0,49)$ de la evaluación general sobre las características tecnológicas del proceso refleja un cumplimiento del $93 \%$ de los requisitos de calidad referidos a un fundente comercial de reconocido prestigio (calificación de 5) [8].

La densidad aparente de los granos de cuasicerámica es de 1,796 $\mathrm{g} / \mathrm{cm}^{3}$ que representa el $74 \%$ de la densidad picnométrica de los granos de la cuasicerámica. Mientras, el índice de consumo de los granos de la cuasicerámica es de 0,69 por 100 g de alambreelectrodo empleado.

\section{Proceso de adsorción de la humedad}

En general, el poder adsorbente de las matrices de los fundentes aglomerados pueden ordenarse según el tipo de adsorbato presente en el proceso de adsorción, pero en la soldadura automática bajo fundente (SAW), la afinidad de la matiz de los fundentes por el vapor de agua es de gran interés, ya que éste puede provocar mermas en las propiedades mecánicas del metal depositado después el proceso de soldadura. Las características polares de la molécula de agua contribuyen a que sea adsorbida con preferencia respecto a los restantes componentes del aire $\left(\mathrm{N}_{2}, \mathrm{O}_{2}\right.$ y otros), que no presentan tan marcado dipolo, por lo que la concurrencias de los mismos por los sitios activos de la superficie de la matriz es relativamente insignificante frente al vapor de agua. Además de la afluencia hacia los sitios activos de la matriz, las moléculas del agua son altamente favorecida debido a que el agua presenta la mayor temperatura crítica y, por tanto, mayor facilidad de licuarse, propiedades que conducen preferentemente a las moléculas de agua a depositarse en la superficie y a su condensación en poros y grietas pequeñas a presiones que están bastante por debajo de la presión de vapor de saturación para una temperatura dada.

Considerando que los granos de la matriz (M) aportan sólo el campo de fuerzas del tipo van der Waals y la superficie geométrica, donde se asentarán las moléculas de agua, entonces estas moléculas formarán una fase adsorbida con características independientes a las del aire seco y las de la matriz. Entonces el proceso de adsorción del agua (A) por el absorbente (M) puede describirse mediante la ecuación $\mathrm{A}+\mathrm{M} \quad \mathrm{AM}+\mathrm{Q}$, donde $\mathrm{Q}$ es el calor que se libera y AM, el complejo adsobato-adsorbente, cuyo estado de equilibrio $(\mathrm{K})$ puede expresarse, a partir de sus concentraciones [ ], de la forma siguiente:

$$
K=\frac{[A M]}{[A \amalg M]}
$$

Generalmente, la adsorción física se acusa a bajas temperaturas y deja de adsorberse a temperaturas relativamente altas. Por tanto, las muestras de granos (entre $+0,25 \mathrm{~mm}$ y $-2,5$ mm) M-n $(\mathrm{n}=1,2,3,4$ y 5) de la cuasicerámica obtenida a 950 ${ }^{\circ} \mathrm{C}$ y $4 \mathrm{~h}$, descrita anteriormente, se calentaron posteriormente en capsulas de porcelana a $450{ }^{\circ} \mathrm{C}$ para eliminar la posible humedad. Las muestras de granos calentadas a $450{ }^{\circ} \mathrm{C}$ se colocaron inmediatamente en una desecadora con acido sulfúrico (98\%) hasta alcanzar la temperatura ambiente. Para obtener las datas experimentales de incremento en masa húmeda en función del tiempo. Las muestras secas se sitúan en una atmósfera de 92,5 $\pm 0,6 \%$ de humedad relativa $(\mathrm{Hr})$ y temperaturas constantes de 293, 298, 303, 308 y $313 \mathrm{~K}$.

La atmósfera experimental se creó artificialmente en una cámara climatizada especialmente diseñada para estos fines [20, 21] . La cámara consta de un sicrómetro acoplado, constituido por dos termómetros de exactitud $0,2{ }^{\circ} \mathrm{C}$ que permiten el registro de las temperaturas húmeda y seca, y mediante la Tabla psicrométrica correspondiente se determina la humedad relativa en su interior. En este caso, el mantenimiento de la humedad requerida se garantiza mediante una solución de $\mathrm{K}_{2} \mathrm{SO}_{4}$ e inyectando y extrayendo vapor de agua, según se requiera, mediante un atomizador y un extractor acoplados al cuerpo de la cámara respectivamente. La atmósfera interior se homogeniza mediante un sistema de ventilación que se encuentra instalado en la cámara y que hace circular el aire a muy baja velocidad. El régimen de temperatura se garantiza mediante una pequeña lámpara infrarroja. La temperatura de la cámara es programada y regulada automáticamente. Las condiciones experimentales de cada ensayo se exponen en la Tabla 8. 
Tabla 8. Condiciones experimentales iniciales de la humectación

\begin{tabular}{|l|c|c|c|c|c|}
\hline \multirow{2}{*}{ Parámetros } & \multicolumn{5}{|c|}{ Muestras de granos $($ de $+0,25 \mathrm{~mm}$ a $-2,5 \mathrm{~mm})$} \\
\cline { 2 - 6 } & $\mathrm{M}-1$ & $\mathrm{M}-2$ & $\mathrm{M}-3$ & $\mathrm{M}-4$ & $\mathrm{M}-5$ \\
\hline $\mathrm{m}\left(\mathrm{t}_{0}\right), \mathrm{g}$ & 182,736 & 180,529 & 181,201 & 179,023 & 178,591 \\
\hline $\begin{array}{l}\mathrm{m}\left(\mathrm{H}_{2} \mathrm{O}\right)_{\mathrm{ab}}, \\
\mathrm{mg}\end{array}$ & 328,3 & 248,9 & 183,7 & 154,9 & 106,4 \\
\hline $\mathrm{T}, \mathrm{K}$ & 293 & 298 & 303 & 305 & 313 \\
\hline $\mathrm{Hr} \%$ & 94 & 93,5 & 92,9 & 91,1 & 91,1 \\
\hline $\mathrm{Hab}, \mathrm{mg} / \mathrm{g}$ & 0,0138 & 0,01874 & 0,03384 & 0,03315 & 0,04423 \\
\hline $\mathrm{Pv}, \mathrm{mb}$ & 15,2 & 29,63 & 39,45 & 51,27 & 67,26 \\
\hline $\begin{array}{l}\mathrm{m}\left(\mathrm{H}_{2} \mathrm{O}\right)=\mathrm{m}\left(\mathrm{t}_{\infty}\right)-\mathrm{m}\left(\mathrm{t}_{\mathrm{o}}\right): \text { vapor absorbido por los granos hasta } \\
\text { la saturación, }\left(\mathrm{t}_{\infty}\right)\end{array}$ \\
\hline
\end{tabular}

Los incrementos porcentuales en masa húmeda de las probetas $(\bar{C}(t))$ se determinan por la expresión:

$C(t)=\left\{\left[m(t)-m\left(t_{0}\right)\right] / m\left(t_{0}\right)\right\} \cdot 100 \%$

en la que $\boldsymbol{m}(\boldsymbol{t})$ es la masa de los granos húmedos en el tiempo $\boldsymbol{t}$ de exposición a la atmósfera dada, la que se mide en una balanza analítica digital de exactitud $\pm 0,1 \mathrm{mg}$ a intervalos de tiempos preferiblemente fijos y $\boldsymbol{m}\left(\boldsymbol{t}_{\boldsymbol{o}}\right)$ es la masa de la muestra en el instante inicial del ensayo (masa del producto seco).

Los ensayos se dan por concluidos cuando la masa húmeda $\boldsymbol{m}(\boldsymbol{t})$ de las muestras no cambia o cambia muy poco, $\boldsymbol{m}\left(\boldsymbol{t}_{\infty}\right)$, es decir, cuando sus incrementos o decrementos están en el orden de exactitud de la balanza.

\subsection{Cinética de la humectación}

Durante la determinación de la cinética de adsorción de la humedad en los granos de la matriz se registraron los cambios de la masa de las muestras en el tiempo y la variación de la masa respecto al tiempo fue calculado tomando datos durante los últimos $600 \mathrm{~s}$. En la medida que $d m / d t$ se aproximaba a cero, la masa de las muestras varía menos y ésta se aproxima a la humedad de equilibrio. Para este estudio se considera un valor de humedad de equilibrio en $d m / d t$ de $7,80 \cdot 10^{-4} \mathrm{mg} / \mathrm{s}$ a $20^{\circ} \mathrm{C}$ y de $1,00 \cdot 10^{-3} \mathrm{mg} / \mathrm{s}$ a $40{ }^{\circ} \mathrm{C}$, como criterio de equilibrio bajo las condiciones experimentales expuestas en la Tabla 8. Los cambios de masa son usados para calcular el contenido de humedad de los granos de matriz en las isotermas (Figura 5). Los cambios de humedad respecto al tiempo fueron almacenados para obtener la cinética de adsorción a cada humedad relativa.

El desarrollo de las isotermas experimentales del proceso de humectación de la matriz surgiere que éstas clasifiquen en las isotermas del tipo I (Figura 5), en las que se insertan las isotermas de adsorción física catalogadas como isotermas de Langmuir entre otras [22, 23].

Los procesos de absorción son utilizados en muchas áreas. Sin embargo, la dinámica general de los procesos es compleja y no bien entendida. Sólo para los sistemas sencillos con isotermas lineales se obtienen soluciones analíticas detalladas. Para modelar los datos experimentales del proceso de absorción de la humedad de los granos de matriz se utilizó los criterios del modelo de Langmuir, que es un modelo teórico que expresa

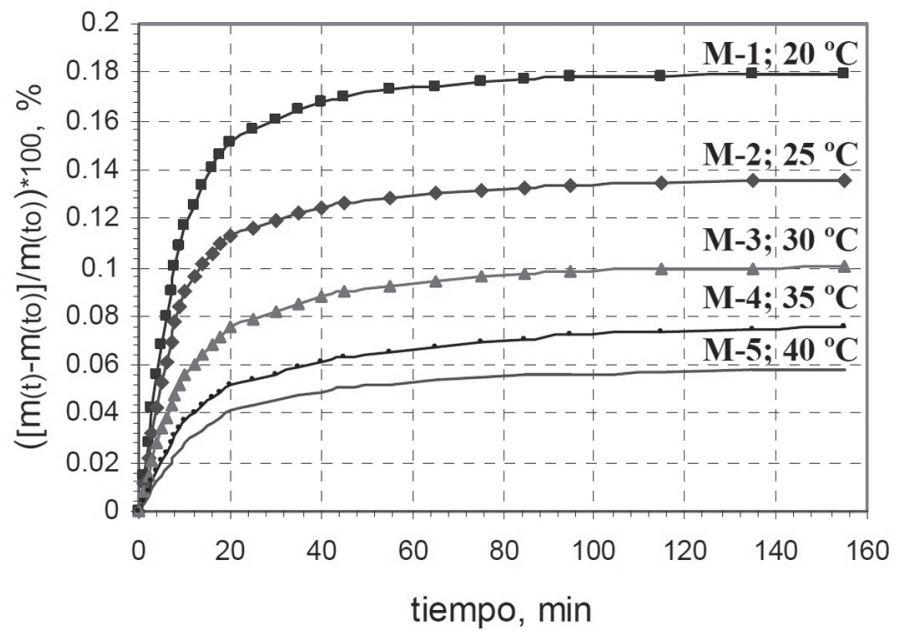

Figura 5. Desarrollo de las isotermas de adsorción de humedad en los granos de matriz a diferentes temperaturas.

la relación entre la concentración del vapor de agua adsorbido y aquella en la fase fluida (gaseosa) a temperatura constante. Este modelo se utiliza para estimar la capacidad máxima de adsorción en el que se fundamenta sobre ciertas suposiciones: a) la superficie es homogénea, b) ambos, el soluto y el solvente ocupan la misma área por molécula adsorbida, es decir por sitio interfacial energéticamente homogéneos, c) no existe interacción colaterales, es decir que la energía de deserción es constante, d) la adsorción máxima corresponde a una capa monomolecular.

La ecuación de Langmuir, en su expresión lineal, puede representarse de la forma siguiente:

$$
\frac{C_{A}}{q_{A}}=\frac{1}{q_{m} \cdot K_{L}}+\frac{C_{A}}{q_{m}}
$$

Donde: $C_{A}$ es la concentración en equilibrio del soluto $\left(\mathrm{H}_{2} \mathrm{O}_{\mathrm{g}}\right)$ en el aire húmedo $(\mathrm{mg} / \mathrm{g}) ; q_{A}$, la cantidad de vapor adsorbido por unidad de masa del adsorbente cuando se alcanza el equilibrio $(\mathrm{mg} / \mathrm{g}) ; K_{L}$ constante de la isoterma de Langmuir $(\mathrm{g} / \mathrm{mg}) ; \mathrm{q}_{\mathrm{m}}$, constante asociada a las características de la superficie $(\mathrm{mg} / \mathrm{g})$.

$K_{L}$ y $q_{m}$ se determinan experimentalmente en una gráfica de $C_{A} / q_{A}$ vs. $C_{A}$, en la que se obtiene una línea recta, siendo $1 / q_{m}$ su pendiente y $1 /\left(q_{m} \cdot K_{L}\right)$ su intersección con las ordenadas (Tabla 9).

En la cámara psicrométrica se somete tres grupos de tres muestras de gramos de matriz (M-n-i, n y i = 1, 2 y 3) con igual granulometría (entre $+0,25 \mathrm{~mm}$ y $-2,5 \mathrm{~mm}$ ) a tres distintas temperaturas y humedades relativas $(\mathrm{Hr}, \%)$ para determinar los parámetros $q_{m}$ y $K_{L}$ de la ecuación de Langmuir (8). Las condiciones y los datos experimentales de las nueve muestras se reportan el la Tabla 8. El valor de $\mathrm{C}_{\mathrm{A}}$ se determina a partir de la ecuación de los gases ideales, $P V=n R T$, $\left(n=m \sqrt{ } / M_{H 2 O}\right)$ :

$$
\frac{m_{V}}{V}=\frac{M_{H 2 O} P_{V}}{R T}
$$

Donde, $m_{v} / V$ es el parámetro $C_{A}$ de la ecuación (8); $M_{H 2 O}$, la masa molecular del agua $(18,02 \mathrm{~g} / \mathrm{mol}) ; P_{V}$, la presión de vapor en equilibrio en atm $(1 \mathrm{mb}=0.0009869 \mathrm{~atm})$; $\mathrm{R}$ la constante 
Tabla 9. Datos experimentales para determinar los parámetros qm y KL de la ecuación de Langmuir

\begin{tabular}{|l|c|c|c|c|c|c|c|c|c|}
\hline \multirow{2}{*}{$\begin{array}{l}\text { Condiciones } \\
\text { experimentales }\end{array}$} & \multicolumn{3}{|c|}{ M-1 } & \multicolumn{3}{c|}{ M-2 } & \multicolumn{3}{c|}{ M-3 } \\
\cline { 2 - 11 } & 1 & 2 & 3 & 1 & 2 & 3 & 1 & 2 & 3 \\
\hline $\mathrm{T}, \mathrm{K}$ & \multicolumn{3}{|c|}{293,15} & \multicolumn{3}{c|}{300,15} & \multicolumn{3}{c|}{313,15} \\
\hline $\mathrm{Hr}, \%$ & 95 & 75 & 65 & 95 & 75 & 65 & 95 & 75 & 65 \\
\hline $\mathrm{m}\left(\mathrm{t}_{\mathrm{o}}\right), \mathrm{g}$ & 177,12 & 169,65 & 172,66 & 181,20 & 167,18 & 175,02 & 178,59 & 170,83 & 173,25 \\
\hline $\mathrm{m}\left(\mathrm{H}_{2} \mathrm{O}\right) \mathrm{ad}, \mathrm{mg}$ & 230,40 & 201,40 & 190,30 & 212,60 & 188,14 & 182,90 & 116,10 & 108,50 & 108,10 \\
\hline $\mathrm{Pv}(\mathrm{vapor}), \mathrm{mb}$ & 22,22 & 17,54 & 15,20 & 33,89 & 26,76 & 23,19 & 70,14 & 55,38 & 47,99 \\
\hline $\mathrm{C}_{\mathrm{A}}, \mathrm{g} / \mathrm{L}$ & 0,0164 & 0,0130 & 0,0112 & 0,0245 & 0,0193 & 0,0167 & 0,0485 & 0,0383 & 0,0332 \\
\hline $\mathrm{q}_{\mathrm{A}}, \mathrm{mg} / \mathrm{g}$ & 1,301 & 1,187 & 1,102 & 1,173 & 1,125 & 1,045 & 0,650 & 0,635 & 0,624 \\
\hline Nomenclatura de la designación de las 9 muestras M-n-i: M-1-1, M-1-2, M-1-3,... M-3-2, M-3-3 \\
\hline
\end{tabular}

universal de los gases $(R=0.0082057 \mathrm{~atm} \cdot L /(K \cdot \mathrm{mol}))$ y $T$, la temperatura en $\mathrm{K}$.

Los resultados experimentales de la adsorción de los granos de la matriz (Tabla 9) se procesaron por regresión lineal obteniéndose las ecuaciones de Langmuir siguientes:

$$
\begin{aligned}
& C_{A} q_{A}=472,06 \cdot C_{A}+4.8547, R^{2}=0,9986 ; 293,15 K \\
& C_{A} q_{A}=638,6 \cdot C_{A}+5.1273, R^{2}=0,9891 ; 300,15 K \\
& C_{A} q_{A}=1399,3 \cdot C_{A}+6.7366, R^{2}=0,9995 ; 313,15 K
\end{aligned}
$$

A partir de las ecuaciones (10), (11) y (12) y para cada condición experimental (Tabla 8 ) se determinaron las constantes de la isoterma de Langmuir $\left(K_{L}\right)$ y las constantes asociadas a las características de la superficie $\left(q_{m}\right)$, las que son reportadas en la Tabla 10.

Tabla 10. Constantes de las isotermas de Langmuir

\begin{tabular}{|l|l|l|l|}
\hline $\mathrm{T}, \mathrm{K}$ & 293,15 & 300,15 & 313,15 \\
\hline$q_{m}$, & $2,118 \cdot 10^{-3}$ & $1,566 \cdot 10^{-3}$ & $7,146 \cdot 10^{-4}$ \\
\hline$K_{L^{\prime}}$ & 97,2377 & 124,549 & 207,7161 \\
\hline
\end{tabular}

\subsection{Termodinámica de la humectación}

$\mathrm{Al}$ interpretar la humectación como un proceso espontáneo $(\Delta \mathrm{G}<0)$ de transferencia de vapor de agua $\mathrm{A}\left(\mathrm{H}_{2} \mathrm{O}_{\mathrm{g}}\right.$, adsorbato $)$ en el aire (tres dimensiones) hacia la superficie de los granos de la matriz $\mathrm{M}$ (adsorbente, dos dimensiones) permite evaluar los cambios de los potenciales termodinámicos, tales como la energía libre de Gibbs $(\Delta \mathrm{G})$, la entropía $(\Delta S)$ y la entalpía $(\Delta \mathrm{H})$. A causa de una disminución de los grados de libertad que sufren las moléculas en esta transferencia, la entropía disminuye $\left(\mathrm{S}_{\mathrm{f}}<\mathrm{S}_{\mathrm{i}} ; \Delta \mathrm{S}<0\right)$, dando como resultado que el proceso sea exotérmico $(\Delta \mathrm{H}<0)$. Según Principio de Le Châtelier, con la disminución de la temperatura experimental y aumento de la presión es favorecida la humectación, pero con el aumento de la temperatura, tal como lo anuncia la expresión $\Delta H=\Delta G+$ $\Delta \mathrm{S} \cdot \mathrm{T}<0$, no indica que el proceso sea más exotérmico [24].
Por lo general los calores de integrales de absorción física de la humedad a temperatura ambiental, son parecidos a su calor de condensación $\left(\Delta \mathrm{H}_{\text {cond }}=9,77 \mathrm{kcal} / \mathrm{mol} \mathrm{a} 25^{\circ} \mathrm{C}[22]\right)$.

A partir de los datos de la Tabla 10 y considerando las expresiones de la energía libre de Gibbs, $\Delta G=\Delta H-\Delta S \cdot T$ y $\Delta G=-n R T \cdot \ln \left(K_{e q}\right)$, pueden calcularse la entalpía $\left(\Delta H_{a d}\right)$, entropía $\left(\Delta S_{a d}\right)$ y energía libre de Gibbs $\left(\Delta G_{a d}\right)$ del proceso de humectación de los granos de la matriz cuasicerámica $\left(950^{\circ} \mathrm{C}\right.$ y 4h). Para el cálculo de estos parámetros del proceso de adsorción se parte del enunciado termodinámico siguiente:

$$
\ln \left(K_{L}\right)=-\frac{\Delta H_{a d}}{R} T^{-1}+\frac{\Delta S_{a d}}{R}
$$

Al graficar los valores reportados en la Tabla 7 en forma de $\ln \left(K_{L}\right)$ vs $1 / T$ se obtiene la línea recta de la Figura 6, que por regresión lineal se obtiene la ecuación siguiente:

$$
\ln \left(K_{L}\right)=-3507,3 \cdot T^{-1}+16,531 ; \quad R^{2-}=0,9981
$$

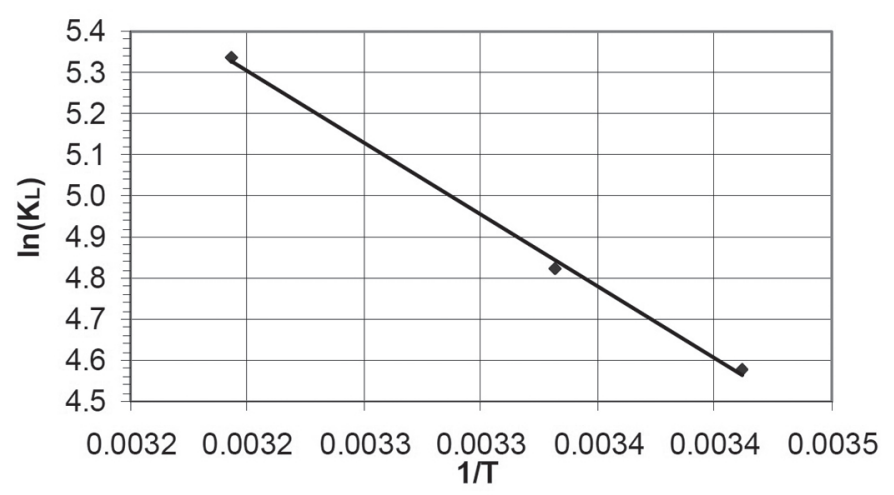

Figura 6. Comportamiento del logaritmo neperiano de la constante de Langmuir $\left(\mathrm{K}_{\mathrm{L}}\right)$ en función de $1 / \mathrm{T}$

A partir de de la comparación de las ecuaciones (13) y (14) se calculan los valores de los parámetros termodinámicos del proceso de humectación: $\Delta H_{a d}=-6,97 \mathrm{kcal} / \mathrm{mol}, \Delta S_{a d}=32,8$ $\mathrm{cal} /(\mathrm{mol} \cdot \mathrm{K})$ y $\Delta G_{a d}=-7,0 \mathrm{kcal} / \mathrm{mol}$. Estos valores están en correspondencia con el orden de los reportados para los calores de condensación del vapor de agua a temperaturas cercanas. 


\section{Conclusiones}

1. La composición química de los minerales zeolita, feldespato y calcita y, esencialmente, la relación $\mathrm{SiO}_{2} / \mathrm{Al}_{2} \mathrm{O}_{3}$ de los dos primeros constituyen aspectos importantes para el diseño de la matriz de fundente aglomerado y el uso del diagrama de fase ternario $\mathrm{SiO}_{2}-\mathrm{Al}_{2} \mathrm{O}_{3}-\mathrm{CaO}$, de los índices de basicidad $\left(\mathrm{IB}_{\mathrm{m}}\right)$ y actividad química $\left(A_{q}\right)$ y la temperatura Temman $\left(\mathrm{T}_{\mathrm{T}}\right)$ conforman las herramientas estrategias para la concepción inicial de la síntesis de la matriz.

2. El comportamiento tecnológico y operacional de la cuasicerámica granulada (entre $+0,25 \mathrm{~mm}$ y $-2,5 \mathrm{~mm}$ ) durante la soldadura se califica de muy bueno $(4,65 \pm 0,49)$ en comparación con un fundente comercial de reconocido prestigio.

3. El criterio cualitativo del grado de amorfismo (GA) obtenido por DRX y la valoración cuantitativa del grado de sinterización (GS) hacen más precisa la selección de la matriz $\left(950^{\circ} \mathrm{C}, 4 \mathrm{~h}\right)$, que, además, presenta un comportamiento químicamente más inerte ante la interacción con el agua, criterios que minimizan la presencia del fenómeno de quimisorción de la humectación en los granos de matriz.

4. El proceso de adsorción de vapor de agua a una humedad casi constante $(\mathrm{Hr}, 96,2 \%)$ a varias temperaturas se manifiesta como isotermas del primer tipo, donde se incluyen las de Langmuir, que se caracterizan por un valor experimental de humedad en $d m / d t$ de $7,80 \cdot 10^{-4} \mathrm{mg} / \mathrm{s}$ a $20{ }^{\circ} \mathrm{C}$ y de $10^{-3} \mathrm{mg} / \mathrm{s}$ a $40{ }^{\circ} \mathrm{C}$ como criterio de equilibrio bajo las condiciones experimentales con que fueron realizadas.

5. La ecuación de Langmuir, $C_{A} / q_{A}=1 /\left(q_{m} \cdot K_{L}\right)+C_{A} / q_{m}$, expresa un acertado comportamiento $\left(\mathrm{R}^{2}=0,9891-0,9995\right)$ del proceso de adsorción al reproducir los datos experimentales obtenidos en la cámara psicrométrica

6. La determinación de las constantes de las isotermas de Langmuir $\left(K_{L}\right)$, a partir de la ecuación lineal, permite utilizar las expresiones de la energía libre de Gibbs, $G=H-S \cdot T$ y $G=-n R T \cdot \ln \left(K_{e q}\right)$, para calcular la entalpía $\left(H_{a d}\right)$, entropía $\left(S_{a d}\right)$ y energía libre de Gibbs $\left(G_{a b}\right)$ del proceso de adsorción, cuyos valores están en correspondencia con los reportados para el proceso de condensación del vapor de agua.

7. Las determinaciones cinéticas y termodinámicas del proceso de humectación de las matrices de fundentes definen el carácter de reversibilidad del proceso y precisan si un simple tratamiento térmico es suficiente para preservar a estos consumibles de soldadura con los niveles de humedad apropiados sin afectar las propiedades físico-mecánicas del cordón de soldadura.

\section{Bibliografía}

1. Probst, R. and H. Herold, Kompendium der Schweisstechnik. 2da ed. Fachbuchreihe Schweisstechnik. Vol. 2. Shweissmetallurgie. 2002, Dusseldorf: DVS-Verlag GmbH. $302 \mathrm{pp}$.

2. Quintana-Puchol, R., L. Perdomo-González, and C.R. Gómez-Pérez, Estrategia sobre el desarrollo de fundentes para la SAW a partir de minerales cubanos. II Encuentro Nacional de Gestión Tecnología, Academia de Ciencias de Cuba, Habana, 1993: p. 55.

3. Kou, S., Welding Metallurgy. 2da ed. 2003, Hoboken, New Jersey.: John Weley \& Sons, INC., Poblication. 465 pp.

4. Quintana-Puchol, R., et al., Concepción Metodológica en la Confección de Matrices Vitreas para Fundentes Aglomerados destinados a la Soldadura Automática, a Partir de Caolín y Dolomita. Revista Soldagem e Inspeçao, 2006. 11(2): p. 8592.

5. Hinz, W., Silicat Lexikon. 1985, Berlín: Akademi-Verlag. $942 \mathrm{pp}$.

6. Lancaster, J.F., The Physics of Welding. 2nd ed. 1986: Pregamon Press. 132pp.

7. Grong, O., Metallurgical Modelling of Welding. 1994, London: The Institute of Material, editorial University Press. Cambrige. 580 pp.

8. Quintana-Puchol, R. and A. Herrera-Artiles, Desarrollo de una matriz fundida obtenida a partir de caolín y dolomita para un fundente aglomerado destinado al recargue por soldadura automática. Revista Soldagem e Inspeçao, 2006. 11(3): p. 156-163.

9. Rosler, H.J., Lehrbuch der Mineralogie. 1983, Leipzig: VEB Deutscher Verlag fur Grundstoffindustrie. (pp.404-409) 833 pp.

10. VDV, Slackenatlas. Verlag Stahleisen M.B.H. 1981, Dusseldorf: Verein Deutsche Eisenhuttenleute Verlag (VDV). 282 pp.

11. Portal-Depestre, G.A., Desarrollo de fundente no-fundido para el relleno de rodillos de buldózeres y piezas que trabajan en condiciones similares. Tesis doctoral in Centro de Investigaciones de Soldadura. 1994, Universidad Central de Las Villas: Santa Clara. p. 121.

12. Potapov, N.N., Materiales para soldar. Tomo 1. Gases protectores y fundentes (en ruso). 1989, Moscú: Machinostroenia ( Construcion de Maquinarias). 544 pp.

13. Tuliani, S.S., T. Boniszewski, and N.F. Faton, Notch toughness of comertial submerged-arc weld metal. Welding and Metal Fabrication, 1969. 37(8 ): p. 327-339.

14. Potapov, N.N., Y.S. Volobuiev, and C.A. .Kurlanov, Sobre la valoración cuantitativa de las propiedades metalúrgicas de los fundentes de soldadura. Revista Construcciones Soldadas, 1980(10): p. 4-16 (en ruso).

15. Giannetto, G., ed. Zeolitas: Características, propiedades y aplicaciones industriales. 1990, Ediciones Innovación Tecnológica: Caracas. pp. 250.

16. Rahaman, M.N., Ceramic processing and sentering. 2001, CD-Copyright, Marcel Dekker: New York. p. 875.

17. Rosler, H.J. and H. Lange, Geochemische Tabellen. 1975, Leipzig: VEB Deutscher Vertrag fur Grundstoffindustrie. p. 375-379.

18. Quintana-Puchol, R., et al., Desarrollo de un Fundente Aglomerado Destinado al Recargue por Soldadura Automática con Matriz Fundida Obtenida a partir de Caolín y Dolomita. Revista Soldagem e Inspeçao, 2006. 11(3): p. 156-163.

19. Gómez-Pérez, C.R., Obtención fundido para la S.A.A.S 
a partir de rocas cubanas. Tesis Doctoral, in Centro de Investigaciones de Soldadura 1995, Universidad Central "Marta Abreu" de Las Villas: Santa Clara. p. 120.

20. Hernández-Ruiz, J.E., et al., Difusión de humedad en mezclas de moldeo para machos de fundición. Boletin de Sociedad Española de Cerámica, 2002. 41(2): p. 41-46.

21. Pino-Rivero, L., et al., Desarrollo de una nueva mezcla autofraguante para machos de fundición. Boletin de Sociedad Española de Cerámica, 2009. 48(2): p. 81-90.

22.Zuara-Nieto, E. and C.I.Beristein-Guevara, Estudio termodinámico y cinético de la adsorción de agua en proteina de suero de leche. Revista Mexicana de Ingeniería Química. Universidad Autónoma Metropolitana-Iztapalapa, D.F., 2007. 6(3): p. 359-365.

23. Sing, K.S.W., et al., Reporting Physisortion Data for Gas/ Solid Systems with Special Reference to the Determination of Surface Area and Porosity. International Union of Pure and Applicad Chemistry and Physical Chemistry División. Commission on Colloid and Surface Chemistry Incluiding Catalysis. Printed in Great Britain, 1985. 57(4): p. 603-619.

24. Colectivo-de-Autores, Chemie Brockhaus ABC. Vol. II. 1985, Leipzig: VEB F.A. Brockhaus. 